\title{
Comparative Safety and Efficacy of Left Atrial Appendage Occlusion with the Watchman Device and Amplatzer Cardiac Plug: Results of the Russian National Registry
}

\author{
Karapet Davtyan, ${ }^{1}$ Georgiy Simonyan, ${ }^{1}$ Arpi Topchyan $\mathbb{D}^{1},{ }^{1}$ Andrey Kalemberg, ${ }^{1}$ \\ Alexander Romanov, ${ }^{2}$ Vitaliy Shabanov, ${ }^{2}$ Dmitriy Lebedev $\left(\mathbb{D},{ }^{3}\right.$ Sergey Gureev, ${ }^{3}$ Yulia Miller, ${ }^{3}$ \\ Evgeniy Merkulov, ${ }^{4}$ Dmitry Pevzner, ${ }^{4}$ Pavel Mozgovoy, ${ }^{5}$ Vladimir Ufimtsev, ${ }^{5}$ \\ Sergey Boytsov, ${ }^{4}$ and Oksana Drapkina ${ }^{1}$
}

${ }^{1}$ National Medical Research Center for Therapy and Preventive Medicine, 101990 Moscow, Russia

${ }^{2}$ E. Meshalkin National Medical Research Center, 630055 Novosibirsk, Russia

${ }^{3}$ Almazov National Medical Research Centre, 197341 Saint Petersburg, Russia

${ }^{4}$ National Medical Research Center of Cardiology, 121552 Moscow, Russia

${ }^{5}$ Clinic №1 at Volgograd State Medical University, 400079 Volgograd, Russia

Correspondence should be addressed to Arpi Topchyan; atopchyan5@gmail.com

Received 25 June 2020; Revised 11 October 2020; Accepted 19 October 2020; Published 9 November 2020

Academic Editor: Gelin Xu

Copyright (c) 2020 Karapet Davtyan et al. This is an open access article distributed under the Creative Commons Attribution License, which permits unrestricted use, distribution, and reproduction in any medium, provided the original work is properly cited.

\begin{abstract}
Purpose. This multicenter, prospective registry evaluated the comparative safety and efficacy of left atrial appendage occlusion (LAAO) using the Watchman device (WD) and the Amplatzer Cardiac Plug (ACP) in patients with nonvalvular atrial fibrillation (NVAF) in real-world clinical practice in Russia. Methods. The study included data from 200 consecutive NVAF patients (66.8 \pm 7.8 years, $44.5 \%$ female, median CHA2DS2VASc 4, median HAS-BLED 3) who had undergone LAAO implantation using WD $(n=108)$ or ACP $(n=92)$ from September 2015 to December 2017 in 5 medical centers in Russia. The primary safety endpoint was the procedure-related major adverse events, and the primary efficacy endpoint was the composite of thromboembolic events, device thrombosis, hemorrhagic events, and unexplained death during the 12-month follow-up. Results. Successful LAAO was performed in all 92 (100\%) patients with ACP and 105 (97.2\%) with WD ( $p=0.053)$. At 12 months, primary safety endpoint occurred in $6.5 \%$ of patients in the ACP group with no events in the WD group (6.5\% vs. $0 \%, p=0.008)$. During the 12 -month follow-up, the primary efficacy endpoint has occurred in $8.3 \%$ of patients in the WD group $(n=9)$ and $1.1 \%$ of patients in the ACP group $(n=1)(p=0.016)$. Conclusions. In this multicenter prospective registry, LAA closure with the WD was associated with significantly higher thromboembolic events rate in NVAF patients. Patients, receiving the ACP, had more procedure-related major adverse events. However, further multicenter studies are necessary to evaluate these findings.
\end{abstract}

\section{Introduction}

It is well known that ischemic stroke is the leading cause of mortality and disability in patients with atrial fibrillation (AF) [1]. Left atrial appendage (LAA) is the source of thrombus formation in more than $90 \%$ of patients with nonvalvular AF (NVAF) [2], and exclusion of LAA from systemic circulation may impede thrombus formation [3]. Several studies demonstrated that transcatheter LAA occlusion (LAAO) could be an alternative to oral anticoagulant therapy (OAC) in NVAF patients at high risk of thromboembolic (TEO) and hemorrhagic events [4].

Present devices for endovascular LAAO are divided into three categories according to the LAA closure principle: "plug," "pacifier," and "ligation" [4]. The plug principle is to obstruct the neck of LAA by the LAAO device lobe or 
umbrella, e.g., the Watchman device (WD) [5]. The pacifier devices have an additional disk to close LAA ostium from the left atrial site in addition to the device lobe/umbrella (Amplatzer Cardiac Plug (ACP), Amplatzer Amulet [6]). Complete ligation of the neck of the LAA is the mechanism of closure with ligation devices (LARIAT) [7].

However, the data on comparative safety and efficacy of LAA closure using WD and ACP are limited to a few single-center studies $[8,9]$.

This registry aimed at assessing the safety and efficacy of the WD compared with ACP for thromboembolic events prevention and adverse events in patients with NVAF in a multicenter study.

\section{Methods}

This multicenter, non-randomized prospective registry included data from 200 consecutive NVAF patients, who undergone LAAO implantation using WD $(n=108$, WD group) or ACP ( $n=92$, ACP group) from September 2015 to December 2017 in 5 medical centers in Russia within the framework of the state catheter-based LAAO program. The WD (Boston Scientific, Natick, Massachusetts) and ACP (St. Jude Medical, St. Paul, MN, USA) were the only LAAO devices approved by the Federal Service on Healthcare Surveillance in Russian Federation. The registry protocol was initially approved by the National Ethics Committee and after that by each center's Local Ethics Committee. Written informed consent was obtained from all patients before enrollment.

The inclusion criteria were age $\geq 18$ years, NVAF, $\mathrm{CH}$ $\mathrm{A}_{2} \mathrm{DS}_{2} \mathrm{VASc} \geq 2$, high risk of hemorrhagic events, and noncompliance with pharmacological OAC therapy. The exclusion criteria were significant mitral valve disease, left ventricular ejection fraction $(\mathrm{LVEF})<35 \%$, the tendency to systemic thrombosis, and severe comorbidities.

Preprocedural transesophageal echocardiography (TEE) was performed to exclude LAA thrombosis and evaluate LAA dimensions. LAAO procedures were carried out under continuous intravenous sedation with propofol or general anaesthesia with endotracheal intubation. Transseptal puncture was performed under the TEE guidance using an $8 \mathrm{Fr}$. transseptal sheath (usually SL0, St. Jude Medical) with the Brockenbrough needle. Intravenous heparin was administered at the time of transseptal puncture (target activated clotting time $\geq 250 \mathrm{~s}$ ). A pigtail catheter was advanced over the 0.035-inch J-tipped guidewire into the LAA, and LAA angiography was performed in several views for better evaluation of LAA anatomy. The device size was selected to be 10-20\% larger than the diameter of the landing zone based on TEE and angiographic measurement of LAA [4]. After that, a stiff J-tipped guidewire was inserted either into the distal LAA or left superior pulmonary vein depending on the operator. The transseptal sheath was exchanged to the appropriate delivery system, and the occluder was advanced via the delivery system to the LAA. After deployment, the device position, compression grade, and the completeness of LAA closure were assessed by both TEE (including colour-flow Doppler) and angiography. Tug test under fluoroscopic and TEE guidance was performed to confirm device stability $[4,6]$.
In two clinics, either WD or ACP was available for LAAO implantation. In the other three clinics, both WD and ACP were used, and device selection depended on operator preferences.

Postprocedural antithrombotic therapy strategy was left to the discretion of doctors.

Patients' baseline characteristics, procedure, and followup data were collected according to established registry protocol. Patients were followed at 45 days and 3, 6, and 12 months after enrollment. Diagnostic TEE was performed at 45 days and six months to assess LAAO device positioning, smoothness of atrial surface, and presence/absence of residual leaks. At each follow-up visit, the data regarding clinical events and healthcare utilization was collected.

The primary safety endpoint included procedure-related major adverse events (pericardial effusion, cardiac tamponade, device embolization, and procedure/device-related death). The primary efficacy endpoint was the composite of TEO, device thrombosis, hemorrhagic events, and unexplained death during the 12-month follow-up. The secondary endpoints were successful LAAO implantation rate and incidence rates of significant ( $\geq 5 \mathrm{~mm}$ ) leakage on 6-month TEE.

2.1. Statistical Analysis. The number of study participants $(n=200) \quad$ was predefined by the government-funded character of the trial. Continuous variables were presented as mean \pm standard deviation (SD), median (Me), and interquartile range (IQR), categorical variables-as frequencies. Comparisons between two groups were performed with the Mann-Whitney $U$ test, chi-square test, and 2-sided Fisher's exact test, as appropriate. A Spearman's correlation test was performed to evaluate the relationship between two methods for measuring LAA size. The Kaplan-Meier analysis with the log-rank test was run to estimate primary endpoints. The Cox proportional hazard model was used to evaluate the effect of prior LAAO procedure experience on the primary endpoints when possible: the hazard ratio (HR) and $95 \%$ confidence intervals (CI) were calculated. A two-tailed $p$ value $\leq 0.05$ was regarded significant. Statistical analysis was performed using Stata v15.0 for Windows (StataCorp., USA).

\section{Results}

3.1. Patients' and Procedural Characteristics. Two hundred patients with NVAF from 5 medical centers, who underwent LAAO implantation using WD $(n=108)$ and ACP $(n=92)$, were consecutively recruited in the study between September 2015 and December 2017. The mean age of the total study participants was $66.8 \pm 7.8$ years; there were slightly fewer females $(44.5 \%)$ than males. No significant differences were observed in age $(66.9 \pm 7.9$ vs. $66.7 \pm 7.6, p=0.795)$ and gender (female $45.4 \%$ vs. $43.5 \%, p=0.795$ ) between the groups. The percentage of patients with concomitant arterial hypertension $(\mathrm{AH})$ was significantly higher in WD group ( $90.7 \%$ vs. $76.4 \%, p=0.006)$, the frequency of congestive heart failure (CHF) (59.3 vs. 80.4, $p=0.001$ ), and prior ischemic stroke (19.4 vs. 32.6, $p=0.033)$-in ACP group. The $\mathrm{CHA}_{2} \mathrm{DS}_{2} \mathrm{VASc}$ score ranged from 2 to 8 (median score 4). The median of HAS-BLED score was 3. More than half of 
the patients were taking Warfarin, but only the third of them achieved the target INR (32.3\%).

Baseline clinical and demographic characteristics of patients are presented in Table 1.

The same operators carried out the LAAO procedures in each clinic. Successful LAA occluder implantation was performed in all 92 (100\%) patients with ACP and 105 (97.2\%) with WD $(p=0.053)$. In these 3 cases, there was a displacement of the WD during tug test despite several attempts of reposition. The mean size of WD was $26.3 \pm 3.8 \mathrm{~mm}$, and the mean ACP size was $24.6 \pm 3.7(p=0.002)$. There was no significant difference in the mean contrast media volume used (155 vs. $150 \mathrm{ml}, p=0.776)$.

3.2. Primary Endpoints. 188 from 200 patients (94\%) completed a 12-month clinical follow-up. One procedure-related death occurred the next day after the procedure. Two patients died during the follow-up period. One of them in the ACP group died in 6 weeks after LAAO implantation. No autopsy was performed; therefore, the exact cause of death was not determined. The other patient in the WD group died in 8 months after the procedure from stomach cancer.

At 12 months, primary safety endpoint occurred in 6.5\% of patients in the ACP group with no events in the WD group (6.5\% vs. $0 \%, p=0.008)$ (Figure 1 ).

In the ACP group, one procedure-related death occurred the next day due to pulmonary trunk perforation with fatal cardiac tamponade, and one patient developed haemotamponade requiring pericardiocentesis. In one case, device migration occurred to the left ventricle the next day, and subsequent endovascular removal of the occluder device was performed. In two patients, device embolization to abdominal aorta was identified on 45-day TEE. In one case, LAAO device was successfully removed via the right femoral artery by the percutaneous procedure. The other patient underwent open aortic surgery with aorto-left femoral bypass. One patient in the ACP group developed intraprocedural right femoral artery trauma by LAAO device sheath requiring urgent surgical intervention (Table 2).

Vascular access site minor complications (hematoma and bleeding), managed conservatively, occurred in one patient from each group. The procedure-related total complication rate was significantly higher in the ACP group compared with the WD group (9.8\% vs. $0.9 \%, p=0.006)$.

At 12 months, the primary efficacy endpoint occurred in $8.3 \%$ of patients in the WD group $(n=9)$ and $1.1 \%$ of patients in the ACP group $(n=1)(p=0.018)$ (Figure 2).

On 45-day TEE, silent thrombus was observed on the WD in three patients, who were treated with dual antiplatelet therapy (DAPT). In all cases, thrombus resolved on Enoxaparin sodium therapy (within 21 days) without any sequelae. TIA/stroke occurred in 5 patients $(6.2 \%)$ in the WD group with no such events in the ACP group. Two patients presenting with stroke were taking DAPT, and the other two were being treated with NOAC and the combination of NOAC and Aspirin, respectively. One patient did not get any antithrombotic medications. Also, one patient in the WD group developed nasal bleeding requiring hospitalization.
3.3. Secondary Endpoints. There was no significant difference in LAAO device implantation success rate $(97.2 \%$ vs. $100 \%$, $p=0.053)$ and significant peridevice leakage $(\geq 5 \mathrm{~mm})$ incidence rate $(3.0 \%$ vs. $0 \%, p=0.059)$ between the WD group and ACP group. In two patients with leak, silent thrombus formation on the atrial side of WD was observed on 45-day TEE. Both thrombus cases were successfully managed with OAC.

3.4. LAAO Procedure Experience-Based Efficacy and Safety. The complications rate between centers ranged from $0 \%$ to $10.3 \%$ depending on the operators' experience. Only three operators had prior experience of catheter-based LAAO procedure with the implantation volume no less than 50 , which was associated with a significantly lower complications rate $(0.8 \%$ vs. $6.2 \%, p=0.04)$. Five in six cases of major procedure-related adverse events occurred in two clinics without prior LAAO experience. Moreover, only ACP was available in one of these two clinics without prior LAAO experience. The frequency of the WD implantation in experienced centers was significantly higher $(74.1 \%$ vs. $23.5 \%, p<0.0001)$. However, there was no significant difference in the primary efficacy endpoint depending on center LAAO procedural volume (HR 1.27 $(95 \%$ CI $0.24-6.6, p=0.78))$.

3.5. Postprocedural Antithrombotic Therapy Strategy. Oral anticoagulation therapy strategy was left to the physicians' discretion. Antithrombotic therapy did not significantly differ from that in the ACP group $(p=0.054)$ (Figure 3$)$. At 12-month follow-up, the rate of compliance with antithrombotic therapy in the WD group was comparable to that in the ACP group (92.5 vs. 92.1\%, $p=0.934$ ). Approximately half of the patients were on DAPT, and third of the patients on anticoagulant therapy (Warfarin or NOAC). $14.6 \%$ of patients were taking a combination of oral anticoagulants with antiplatelets, and $2.0 \%$ of patients were taking injective anticoagulants (Enoxaparin sodium, Nadroparin calcium).

\section{Discussion}

We evaluated the safety and efficacy of the two most frequently used LAAO devices in Russia (the WD and ACP) in real-world clinical practice. The major findings of this study were that ACP implantation was associated with greater major complications' rate and the use of WD-with higher device thrombus formation/TIA/stroke rate.

The observed major adverse event rate in the ACP group $(6.5 \%)$ was higher than that reported in the large multicenter trial with the Amplatzer Cardiac Plug $(n=1047)$ by Tzikas et al. (4.97\%) [10]. Such high rate is most likely related to the concept of the learning curve $[11,12]$. This governmentfunded catheter-based LAAO program in Russia started in September 2015, and a certain lack of experience existed at that time; two of five clinics had no previous experience of LAAO procedures. Moreover, the frequency of the WD implantation in experienced centers was significantly higher. In the study, conducted by Cruz-Gonzalez et al. [11] to assess the significance of the learning curve in LAAO, the complication rate decreases from $9 \%$ to $0 \%$ with operator experience. 
TABLE 1: Patients characteristics.

\begin{tabular}{|c|c|c|c|c|}
\hline & Total & WD & $\mathrm{ACP}$ & $p$ value \\
\hline Age (years) & $66.8 \pm 7.8$ & $66.9 \pm 7.9$ & $66.7 \pm 7.6$ & 0.795 \\
\hline Female gender $\%(n)$ & $44.5(89)$ & $45.4(49)$ & $43.5(40)$ & 0.788 \\
\hline Permanent AF \% $(n)$ & $46.5(93)$ & $50(54)$ & $42.4(39)$ & 0.539 \\
\hline $\mathrm{AH} \%(n)$ & $84.3(166)$ & $90.7(98)$ & $76.4(68)$ & 0.006 \\
\hline $\mathrm{DM} \%(n)$ & $21(48)$ & $25.9(28)$ & $21.7(20)$ & 0.490 \\
\hline Prior MI \% $(n)$ & $18.1(36)$ & $19.6(21)$ & $16.3(15)$ & 0.544 \\
\hline CHF 2-3 NYHA \% $(n)$ & $69.0(138)$ & $59.3(64)$ & $80.4(74)$ & 0.001 \\
\hline Prior ischemic stroke $\%(n)$ & $25.5(51)$ & $19.4(21)$ & $32.6(30)$ & 0.033 \\
\hline Prior hemorrhagic stroke $\%(n)$ & $4(8)$ & $4.6(5)$ & $3.3(3)$ & 0.622 \\
\hline TIA \% $(n)$ & $6(12)$ & $6.5(7)$ & $5.4(5)$ & 0.756 \\
\hline Neurologic deficiency \% $(n)$ & $17.1(34)$ & $13.1(14)$ & $21.7(20)$ & 0.106 \\
\hline Major bleeding \% $(n)$ & $39.4(78)$ & $33.3(36)$ & $46.7(42)$ & 0.056 \\
\hline Carotid artery stenosis $\%(n)$ & $6(12)$ & $3.7(4)$ & $8.7(8)$ & 0.138 \\
\hline Hepatic dysfunction \% $(n)$ & $2.0(4)$ & $0.9(1)$ & $3.3(3)$ & 0.337 \\
\hline Interatrial shunting $\%(n)$ & 9.7 (19) & $12.0(13)$ & $6.8(6)$ & 0.219 \\
\hline Alcohol abuse \% (n) & $1.0(2)$ & $0.9(1)$ & $1.1(1)$ & 0.909 \\
\hline $\mathrm{OAC} \%(n)$ & & & & 0.198 \\
\hline Warfarin & $62.6(124)$ & $66.7(72)$ & $57.8(52)$ & \\
\hline NOAC & $37.4(74)$ & $33.3(36)$ & $42.2(38)$ & \\
\hline Labile INR \% ( $n)$ & $67.7(84)$ & $65.3(47)$ & $71.1(37)$ & 0.490 \\
\hline $\mathrm{CHA}_{2} \mathrm{DS}_{2}$ VASc score & $4(3-5)$ & $3.5(3-5)$ & $4(3-5)$ & 0.193 \\
\hline HAS-BLED score & $3(3-3)$ & $3(2-3)$ & $3(3-3)$ & 0.348 \\
\hline LA size (mm) & $47(42-52)$ & $47(42-51.5)$ & $47(41-52)$ & 0.735 \\
\hline LVEF (\%) & $57(51-61)$ & $58(52-60)$ & $55(50-63,5)$ & 0.325 \\
\hline
\end{tabular}

Values are presented as mean \pm standard deviation, $\%(n)$, or median (interquartile range). AF, atrial fibrillation; AH, arterial hypertension; DM, diabetes mellitus; MI, myocardial infarction; CHF, congestive heart failure; TIA, transient ischemic attack; OAC, oral anticoagulant therapy; NOAC, non-Vitamin K antagonist oral anticoagulants; INR, international normalized ratio; LA, left atrium; LVEF, left ventricular ejection fraction.
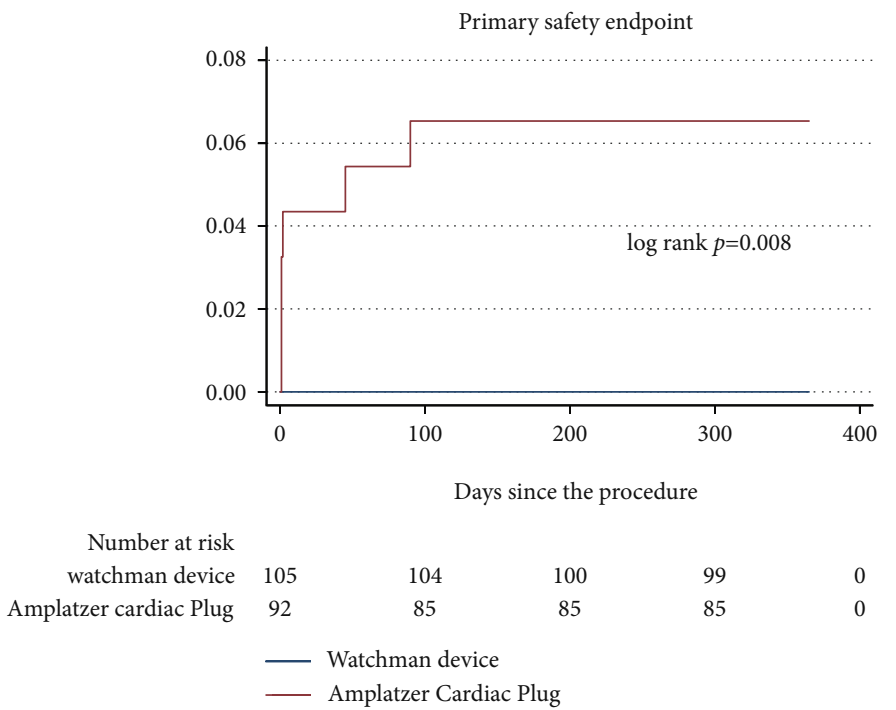

FIgURE 1: Major LAAO-related complications rate using the Watchman device and Amplatzer Cardiac Plug. 
TABLE 2: Major complications of LAAO procedure.

\begin{tabular}{lcc}
\hline Complication type & Number & Incident/detection time \\
\hline Fatal pulmonary trunk perforation & 1 & 1 day \\
Haemotamponade & 1 & 0 day \\
Device migration to the left ventricle & 1 & 1 day \\
Device migration to the aorta & 2 & 45 day \\
Right femoral artery trauma & 1 & 0 day \\
\hline
\end{tabular}

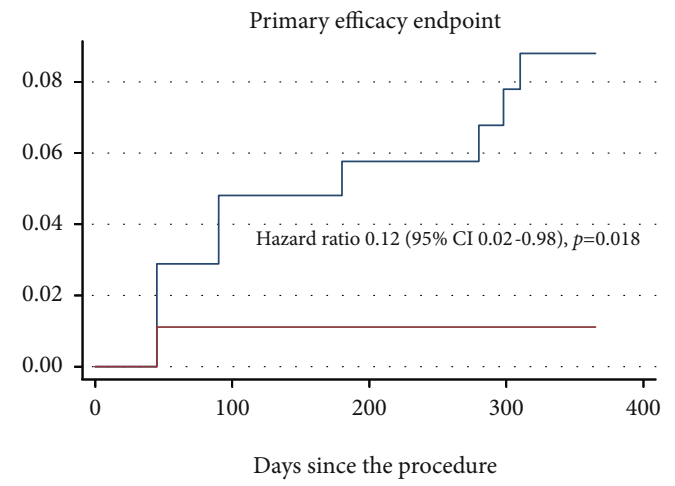

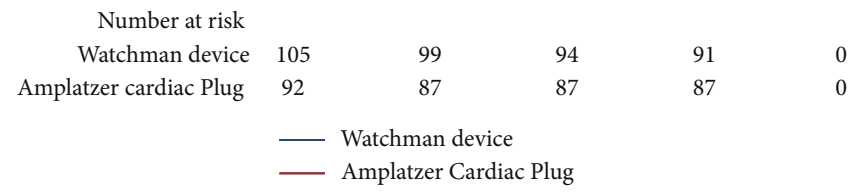

FIgURE 2: Primary efficacy endpoint of LAAO using Watchman device and Amplatzer Cardiac Plug.

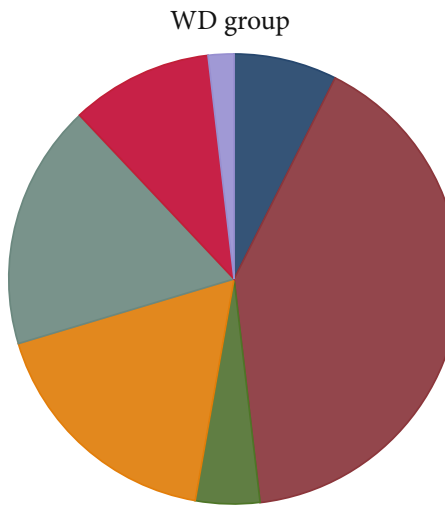

No theraphy

DAPT+Warfarin

NOAC

Other (Enoxaparin sodium, Nadroparin calcium)

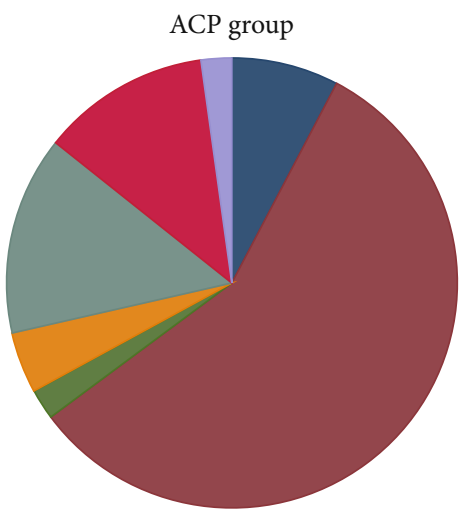

DAPT

Warfarin

$\mathrm{NOAC}+$ Aspirin

FIgURE 3: Antithrombotic therapy strategy after LAAO procedure. DAPT, dual antiplatelet therapy; NOAC, non-Vitamin K antagonist oral anticoagulants.

Moreover, the authors noticed that initial experience of LAAO using ACP also translated into better safety and efficacy outcomes for the WD [11]. The absence of additional disk in WD perhaps simplified the implantation procedure using the WD and therefore the complication rate [12]. In our study, no major adverse events occurred in the WD group, which correlated well with data from the EWOLUTION registry [13]. The analysis of two European real-world registry data, performed by Kleinecke et al. [14] reported a comparable periprocedural major complications rate for the WD and 
Amplatzer group devices ( $4.1 \%$ vs. $6.0 \%, p=0.32)$. A recent meta-analysis of six studies also confirmed the comparable safety outcomes for the WD and Amplatzer groups [15].

Accurate LAAO device size selection is crucial to prevent significant peridevice leakage, which seems to be more common for the WD. In our study on 6-month TEE, major residual leakage was observed in 3 patients. Two of them developed devices' thrombi, fortunately, resolved without any clinical sequelae.

Data regarding the clinical efficacy of LAAO procedure for preventing stroke/TIA correlated strongly with respective studies for the WD [16] and ACP [10]. Tzikas et al. [10] reported the rate of $3.5 \%$ of TIA/stroke/cardiovascular death for an average 13-month follow-up period. In our study, sudden death occurred in 1 patient (1.1\%). Although the number of patients with prior stroke history was significantly higher in the ACP group, there were no clinically significant TIA/stroke episodes in this group. The possible explanation of such findings is the results of a study, conducted by Litwinowicz et al. [17]. The authors evaluated the longterm efficacy of LAAO procedure in primary and secondary stroke prevention and reported comparable efficacy outcome during a mean follow-up of 50 months. The $6.2 \%$ rate of TIA/stroke in the WD group, observed in our study, correlated well with data from the PREVAIL trial (6.4\%) [16] and the aforementioned European registries (6.4\%) [14].

There was no significant difference in comparative efficacy and safety outcomes of LAA closure with WD versus ACP presented by Figini et al. [9]. Peridevice leak incidence was the only significant difference between the groups (WD $18 \%$ vs. ACP $6.3 \%, p=0.037$ ). Such a high incidence of the leak in the WD group was due to cutoff value $>3 \mathrm{~mm}$ for the severe residual leak. These data correlated well with the results of the aforementioned meta-analysis [15]. The rate of total peridevice leakage was significantly higher in the $\mathrm{WD}$ group $(\log \mathrm{OR}=1.32 ; 95 \% \mathrm{CI}=0.76$ to $1.87 ; p<0.01)$. On the other hand, the rate of significant peridevice leakage did not differ significantly between groups. All-cause mortality, cardiovascular mortality, and TIA/stroke rates were also comparable between the WD and Amplatzer device groups [15]. Direct comparison of three devices, the WD, ACP/Amulet, and LAmbre, performed in China, also did not reveal any statistically significant differences in procedure-related events and long-term follow-up outcomes [8]. However, the Amplatzer group included ACP and Amulet devices, which may lead to certain misinterpretation. LAAO procedure performed in a single center by more experienced operators might be the possible causes of such discrepancies between our results and reported data.

\section{Limitations}

This study is a retrospective analysis of prospectively collected registry data from a limited number of study participants. Also, the interpretation of the data was limited due to the small number of events. The device selection depended on operators' discretion and device availability: in two clinics, either the WD or ACP was available. Postprocedural antithrombotic therapy strategy was left to the discretion of treat- ing physicians, which impedes the analysis of postprocedural antithrombotic therapy. However, this is the first study based on the multicenter prospective registry providing real-world experience with LAAO device.

\section{Conclusion}

In this multicenter prospective registry, LAA closure with the WD was associated with significantly higher thromboembolic events rate in NVAF patients. Patients, receiving the ACP, had more procedure-related major adverse events. However, further randomized multicenter studies are necessary to evaluate these findings.

\section{Data Availability}

The data that support the findings of this study are available from the corresponding author, upon reasonable request.

\section{Conflicts of Interest}

Karapet Davtyan serves as a proctor for Medtronic and Abbott. Andrey Kalemberg is a consultant for Abbott. Arpi Topchyan has received a speaker honorarium from Medtronic. Dmitriy Lebedev has received a speaker honorarium from Medtronic and Biosense Webster. The remaining authors have no conflicts of interest to declare.

\section{Acknowledgments}

The trial was supported by the Ministry of Health of the Russian Federation.

\section{References}

[1] E. J. Benjamin, P. Muntner, A. Alonso et al., "Heart Disease and Stroke Statistics-2019 update: a report from the American Heart Association," Circulation, vol. 139, no. 10, pp. e56-e528, 2019.

[2] N. M. Al-Saady, O. A. Obel, and A. J. Camm, "Left atrial appendage: structure, function, and role in thromboembolism," Heart, vol. 82, no. 5, pp. 547-554, 1999.

[3] O. Alli, S. Doshi, S. Kar et al., "Quality of life assessment in the randomized PROTECT AF (percutaneous closure of the left atrial appendage versus warfarin therapy for prevention of stroke in patients with atrial fibrillation) trial of patients at risk for stroke with nonvalvular atrial Fibrillation," Journal of the American College of Cardiology, vol. 61, no. 17, pp. 17901798, 2013.

[4] M. Glikson, R. Wolff, G. Hindricks et al., "EHRA/EAPCI expert consensus statement on catheter-based left atrial appendage occlusion - an update," Europace, vol. 22, no. 2, p. $184,2020$.

[5] M. J. Price, "The WATCHMAN left atrial appendage closure device: technical considerations and procedural approach," Interventional Cardiology Clinics, vol. 7, no. 2, pp. 201-212, 2018.

[6] S. Gloekler, S. Shakir, J. Doblies et al., "Early results of first versus second generation Amplatzer occluders for left atrial appendage closure in patients with atrial fibrillation," Clinical Research in Cardiology, vol. 104, no. 8, pp. 656-665, 2015. 
[7] K. Bartus, F. T. Han, J. Bednarek et al., "Percutaneous left atrial appendage suture ligation using the lariat device in patients with atrial fibrillation: initial clinical experience," Journal of the American College of Cardiology, vol. 62, no. 2, pp. 108118, 2013.

[8] G. Cheung, K. So, C. Chan et al., "Comparison of three left atrial appendage occlusion devices for stroke prevention in patients with non-valvular atrial fibrillation: a single-centre seven-year experience with WATCHMAN, AMPLATZER Cardiac Plug/Amulet, LAmbre," AsiaIntervention, vol. 5, pp. 57-63, 2019, 1 .

[9] F. Figini, P. Mazzone, D. Regazzoli et al., "Left atrial appendage closure: a single center experience and comparison of two contemporary devices," Catheterization and Cardiovascular Interventions, vol. 89, no. 4, pp. 763-772, 2017.

[10] A. Tzikas, S. Shakir, S. Gafoor et al., "Left atrial appendage occlusion for stroke prevention in atrial fibrillation: multicentre experience with the AMPLATZER Cardiac Plug," EuroIntervention, vol. 11, no. 10, pp. 1170-1179, 2016.

[11] I. Cruz-Gonzalez, A. Perez-Rivera, R. Lopez-Jimenez et al., "Significance of the learning curve in left atrial appendage occlusion with two different devices," Catheterization and Cardiovascular Interventions, vol. 83, no. 4, pp. 642-646, 2014.

[12] J. Ledwoch, C. Krollmann, S. Staubach, M. Hug, H. Strohm, and H. Mudra, "Learning curve assessment for percutaneous left atrial appendage closure with the WATCHMAN Occluder," Journal of Interventional Cardiology, vol. 29, no. 4, pp. 393-399, 2016.

[13] L. V. A. Boersma, B. Schmidt, T. R. Betts et al., "Implant success and safety of left atrial appendage closure with the WATCHMAN device: periprocedural outcomes from the EWOLUTION registry," European Heart Journal, vol. 37, no. 31, pp. 2465-2474, 2016.

[14] C. Kleinecke, J. Yu, P. Neef et al., "Clinical outcomes of Watchman vs. Amplatzer occluders for left atrial appendage closure (WATCH at LAAC)," Europace, vol. 22, no. 6, pp. 916-923, 2020.

[15] I. Basu Ray, D. Khanra, S. Shah et al., "Meta-analysis comparing WatchmanTM and Amplatzer devices for stroke prevention in atrial fibrillation," Frontiers in Cardiovascular Medicine, vol. 7, pp. 1-8, 2020.

[16] D. R. Holmes Jr., S. Kar, M. J. Price et al., "Prospective randomized evaluation of the watchman left atrial appendage closure device in patients with atrial fibrillation versus long-term warfarin therapy: the PREVAIL trial," Journal of the American College of Cardiology, vol. 64, no. 1, pp. 1-12, 2014.

[17] R. Litwinowicz, M. Bartus, M. Malec-Litwinowicz, M. Michalski, K. Banaszkiewicz, and B. Kapelak, "Left atrial appendage occlusion for secondary stroke prevention in patients with atrial fibrillation: long-term results," Cerebrovascular Diseases, vol. 47, no. 3-4, pp. 188-195, 2019. 